\title{
Geração de Curvas IDFs para Cenários Projetados na Cidade de Porto Alegre/RS
}

\author{
Adriana Burin Weschenfelder ${ }^{1}$ (D), Eliana Veleda Klering ${ }^{1}$, Rita de Cássia Marques Alves ${ }^{1}$, \\ Éber José de Andrade Pinto ${ }^{2}$ \\ ${ }^{1}$ Centro Estadual de Pesquisa em Sensoriamento Remoto e Meteorologia, \\ Universidade Federal do Rio Grande do Sul, Porto Alegre, RS , Brasil. \\ ${ }^{2}$ Departamento de Engenharia Hidráulica da Universidade Federal de Minas Gerais, \\ Belo Horizonte, MG, Brasil.
}

Recebido em: 14 de Novembro de 2017 - Aceito em: 3 de Janeiro de 2018

\begin{abstract}
Resumo
$\mathrm{O}$ aumento na frequência de eventos extremos associados às alterações climáticas podem modificar as curvas IDFs existentes. A metodologia para definição de curvas IDF considerando cenários futuros consistiu em calibrar o modelo estocástico para gerar séries sintéticas diárias de precipitação com base em dados observados do município de Porto Alegre e gerar séries sintéticas perturbadas a partir de seis MCGs, três cenários A1B, A2 e B1 para os períodos de 2046 a 2065 e 2080 a 2099. Na elaboração das IDFs foram desagregados os dados sintéticos diários, tanto para a linha de base (1974 a 2014), quanto para os cenários projetados, utilizando as relações de desagregação da IDF atual de Porto Alegre. Os resultados obtidos após a calibração indicaram ser o modelo estocástico utilizado eficiente na representação dos dados local. Os resultados encontrados para as projeções no primeiro período indicam que as simulações dos modelos são razoavelmente aplicáveis, sugerindo a utilização da curva IDF atual com uma majoração de 10 a $20 \%$ no dimensionamento de obras hidráulicas. Já para o segundo período de projeção em função da grande variabilidade nos resultados encontrados para os diferentes modelos não há confiança na aplicabilidade destas projeções.
\end{abstract}

Palavras-chave: curvas de intensidade, duração e frequência, impactos mudanças climáticos, modelos de circulação global.

\section{Generation of IDF Curves for Projected Scenarios in the City of Porto Alegre/RS}

\begin{abstract}
The increase in the frequency of extreme events associated with climate change can modify the existing IDF curves. The methodology for defining IDF curves considering future scenarios was to calibrate the stochastic model to generate daily synthetic precipitation series based on observed data from the city of Porto Alegre and to generate perturbed synthetic series from six MCGs, three scenarios A1B, A2 and B1 for the periods 2046 to 2065 and 2080 to 2099. In the elaboration of the IDFs, the daily synthetic data were disaggregated, both for the baseline (1974 to 2014) and for the projected scenarios, using the disaggregation relationships of the current IDF of Porto Alegre. The results obtained after the calibration indicated that the stochastic model used was efficient in the local data representation. The results for the projections in the first period indicate that the simulations of the models are reasonably applicable, suggesting the use of the current IDF curve with a 10 to $20 \%$ increase in the design of hydraulic works. Already in the second projection period due to the great variability in the results found for the different models, there is no confidence in the applicability of these projections
\end{abstract}

Keywords: intensity, duration and frequency curves, climate change impacts, global circulation models.

Autor de correspondência: Adriana Burin Weschenfelder, adriana.burin@gmail.com. 


\section{Introdução}

Alterações na precipitação pluvial aumentam a complexidade na gestão dos recursos hídricos e os efeitos sobre a infraestrutura hídrica. As alterações climáticas são cada vez mais evidentes e o aumento na frequência de eventos extremos de precipitação, associados às instabilidades atmosféricas, pode levar a sobrecarga dos sistemas de escoamento e causar alagamentos e por vezes provocar inundações. Dentro deste contexto, se torna essencial à utilização de curvas IDFs projetadas para diferentes cenários climáticos, a fim de melhorar as obras hidráulicas existentes.

$\mathrm{O}$ aumento da frequência de noites e dias quentes e, consequentemente, a diminuição de noites e dias frios contribuem para elevação da temperatura e aumento da capacidade do ar em reter vapor d'água, que pode contribuir com o aumento na intensidade dos eventos de precipitação e na frequência de dias com precipitações intensas no sudeste da América do Sul (Marengo, 2009).

No Brasil, conforme descrito pelo Painel Brasileiro de Mudanças Climáticas (PBMC, 2014), vem se observando tendência de aumento de precipitação na região sul. No Estado do Rio Grande do Sul observou-se tendência de aumento dos totais pluviométricos e do número de dias com precipitação pluvial, ao longo das últimas décadas, tanto no verão quanto nas estações de transição primavera e outono (Berlato e Cordeiro, 2017).

A tendência de aumento na precipitação, relacionada à maior intensidade e ao maior número de dias com precipitação, leva a prejuízos tanto de ordem econômica como social. No caso da cidade de Porto Alegre, na região sul, de acordo com Reboita (2012) o regime de precipitação é bem distribuído ao longo do ano e é ocasionalmente afetada por eventos extremos de precipitação pluvial. O aumento na intensidade da precipitação compromete a capacidade de armazenamento e a eficácia no escoamento das vazões pelos sistemas de controle urbanos existentes (Todeschini, 2012).

Como maior parte da superfície da área urbana de Porto Alegre é impermeabilizada e há relação direta desta com o escoamento superficial das águas pluviais, uma melhor caracterização das tendências de precipitação, feita através de análises estatísticas, permitem estabelecer a relação entre a intensidade e a duração destes eventos e a probabilidade deles ocorrerem novamente.

Bertoni e Tucci (2004) colocam que para o dimensionamento de obras hidráulicas, entre elas, sistemas de drenagem, galerias pluviais e dimensionamento de bueiros é preciso conhecer intensidade duração e frequência das precipitações máximas. A representação destas pode ser feita por curvas de Intensidade, Duração e Frequência (IDF).

No Brasil, Pfaffstetter (1957) realizou um estudo pioneiro nesta área que reuniu registros pluviográficos de
98 postos do Serviço de Meteorologia do Ministério da Agricultura (atual Instituto Nacional de Meteorologia). Este trabalho ainda hoje é utilizado como referência para projetos de dimensionamento de obras hidráulicas.

$\mathrm{Na}$ cidade de Porto Alegre, entre outros estudos, podemos citar o estudo realizado por Benfica (1999). A autora utilizou a série de dados do $8^{\circ}$ DISME/INMET, no período de 1974 a 1998. Para tanto foram selecionados os maiores eventos anuais ocorridos nas durações de 5, 10, $15,30,60,120,240,360,720,1080$ e 1440 minutos. A distribuição estatística que melhor se adequou após o cálculo dos parâmetros das distribuições pelos métodos dos momentos e da máxima verossimilhança foi a de Gumbel.

Weschenfelder et al. (2015) definiu novas curvas IDF para Porto Alegre. As equações definidas correspondem à atualização da equação estabelecida por Benfica (1999) e recomenda-se sua utilização nas regiões de Porto Alegre sugeridas no Plano Diretor de Drenagem Urbana do Município de Porto Alegre (Universidade Federal do Rio Grande do Sul, 2002). A série de dados do $8^{\circ}$ DISME/ INMET compreendeu o período de 1974 a 2014. Foram selecionados os maiores eventos anuais ocorridos nas durações de $5,10,15,30,45,60,120,180,240,480,840$ e 1440 minutos. A distribuição de frequência ajustada aos dados foi a Exponencial, com os parâmetros calculados pelo método dos momentos-L.

Em muito locais dados de precipitação em intervalos menores, durações entre 5 minutos e 24 horas, não existem, são encontrados apenas informações de dados diários. Para construir IDFs nestes locais é necessário realizar a desagregação dos dados diários em sub-diários.

Chen (1983) apresenta uma fórmula de intensidade duração e frequência para qualquer local nos Estados Unidos. Para obtenção da equação são requeridas alturas de precipitação com tempo de retorno de 10 anos nas durações de 1 hora e 24 horas e tempo de retorno de 100 anos na duração de 1 hora. $\mathrm{O}$ autor retoma trabalhos anteriores de Chen (1975,1976 apud CHEN 1983) que colocam que a relação entre alturas de precipitação ocorridas nas durações de 1 hora e 24 horas, independente do tempo de retorno, variam entre 10 e $60 \%$ dependendo da localização. O autor também compara a equação desenvolvida com a apresentada por Bell (1969 apud Chen 1983) que utiliza somente tempo de retorno de 10 anos e durações de 1 hora e coloca que embora as equações sejam muito parecidas a de Bell é limitada na representação das variações geográficas da precipitação.

Blodgett (1996) calculou os índices de altura de chuva por duração, desagregando os dados diários de precipitação em intervalos de 1, 2, 3, 4, 6, 12 e 18 horas. Os incrementos de precipitação utilizados foram de 5 minutos. Os autores adotaram a relação entre o intervalo verdadeiro de ocorrência de precipitação (24 horas) e o acumulado de 1 dia como 1,13 independente do tempo de recorrência. Segundo Miller et al. (1973) o valor de 1,13, 
definido no Atlas de precipitação e frequência elaborado para várias cidades do oeste dos Estados Unidos, mais especificamente na Califórnia, representa um valor médio.

Back et al. (2012) determinou relações de desagregação para diferentes locais no estado de Santa Catarina para durações entre $5 \mathrm{~min}$ e 24 horas. $\mathrm{O}$ autor chegou a diferentes relações médias para as relações entre 24horas/ 1 dia que variaram entre 1,11 e 1,24. Occhipinti e Santos (1966 apud BACK, 2012) utilizando séries de dados no período de 1928 a 1965 em São Paulo observaram esta relação variando de 1,13 a 1,15 com média de 1,14 , valor recomendado pela Companhia Ambiental do Estado de São Paulo (CETESB).

Pinto (2013) apresenta uma metodologia para definição de uma equação IDF com séries de máximos anuais e série de máximos parcial, quando há disponibilidade de registros contínuos e, quando não há disponibilidade destes registros a definição da IDF é feita por desagregação dos dados diários acumulados em durações menores.

Os estudos de drenagem utilizados como referência nos projetos de dimensionamento de obras hidráulicas, em geral, levam em consideração somente os dados históricos de precipitação obtidos através de registros contínuos ou acumulados.

Soluções específicas ligadas a tipos específicos de ocorrências extremas, conforme Blakely (2007) podem revelar-se ineficazes em suportar os efeitos negativos das alterações climáticas na maioria das cidades. Segundo o autor, embora as cidades tenham estruturas de mitigação, tais como reservatórios de controle de cheias, bacias de retenção de inundação e obras de proteção como diques entre outras, estas precauções não consideram os riscos potenciais que as tendências atuais de mudanças climáticas podem trazer para uma cidade.

Alguns autores tem buscado incorporar alterações previstas pelos MCG, como o trabalho de Sandink et al. (2016) no qual os autores tentando auxiliar na tomada de decisão, desenvolveram uma ferramenta computadorizada para a construção de curvas IDFs incorporando mudanças climáticas. Os autores destacam os impactos dos eventos extremos em vários municípios urbanos Canadenses. No Canadá assim como é feito no Brasil, as curvas IDF são utilizadas na gestão dos recursos hídricos, e desenvolvidas assumindo estacionariedade nos dados, o que não leva em conta as mudanças climáticas. De acordo com os autores a ferramenta computacional desenvolvida permite aos usuários escolher entre 567 estações pré-carregadas, contendo pelo menos 10 anos de dados históricos observados, também podem ser introduzidos dados atualizados na construção das curvas IDF para cenários de projeção de 22 CGM's do AR5 para períodos entre 2006 e 2100.

A avaliação do impacto de mudanças climáticas em curvas IDF na cidade de São Paulo foi apresentada por Schardong e Simonovic (2013). Os autores utilizaram um modelo estocástico para gerar séries de precipitação que representem cenários futuros, mas com as mesmas características, média, desvio padrão e distribuição temporal da série observada. Os cenários futuros foram gerados com as estimativas de modelos de circulação global (GCMs), simulados para o IPCC (Intergovernamental Panel on Climate Change) AR4, um de alta emissão de gases de efeito estufa A2 e um de baixa emissão B1, projetados para os períodos de 2046-2065 e 2081-2100. Os autores ajustaram curvas IDF aos dados históricos observados e as séries geradas para os diferentes períodos e cenários climáticos. Os autores encontram um aumento de $4 \%$ a $26 \%$ na precipitação considerando IDF ajustada aos cenários em relação à IDF ajustada ao período histórico utilizado (1961 a 1997).

O desenvolvimento de cenários climáticos futuros em escala local a partir de mudanças climáticas em larga escala, simuladas por MCG, pode ser realizado por geradores estocástico do clima. (Qian et al., 2005).

Um gerador estocástico bastante utilizado é o Long Ashton Research Station - Weather Generator - LARSWG (Semenov e Barrow, 1997) que permite a simulação de dados meteorológicos em um único local, nas condições climáticas atuais e futuras. (Racsko et al., $1991 \mathrm{e}$ Semenov e Barrow, 2002). Cabe salientar que, para avaliar o impacto sobre as curvas IDFs é necessário transformar os dados sintéticos diários obtidos com o gerador estocástico em sub-diários utilizando relações de desagregação, assim como é feito para locais sem disponibilidade de dados sub-diários.

Ao levarmos em consideração as projeções de mudanças climáticas e considerada a probabilidade de aumento da incidência de eventos extremos, visou-se neste estudo: avaliar a capacidade de representação das precipitações em Porto Alegre, utilizando o modelo LARSWG; desagregar os dados diários sintéticos de precipitação, obtidos com o modelo de LARS-WG para linha de base (1974 a 2014), em sub-diários, utilizando as relações de desagregação fornecidas pela IDF definida por Weschenfelder et al.(2015); avaliar o impacto das de alterações climáticas através das projeções nos cenários A1B, A2 e B1 sobre as curvas de Intensidade, Duração e Frequência (IDF), construídas após a desagregação dos dados sintéticos diários obtidos para cada uma das projeções.

\section{Descrição do Modelo Gerador de Séries Sintéticas de Precipitação Diária}

O LARS-WG produz séries sintéticas diárias de temperaturas máxima e mínima, precipitação e radiação solar. Os dados de entrada do gerador são séries temporais diárias de precipitação $(\mathrm{mm})$, temperaturas máxima e mínima $\left({ }^{\circ} \mathrm{C}\right)$ e radiação solar $\left(\mathrm{MJ} \cdot \mathrm{m}^{-2} \cdot \mathrm{dia}^{-1}\right)$. Os dados de número de horas de insolação podem ser utilizados como alternativa aos dados de radiação solar. Neste caso a 
radiação solar é estimada utilizando abordagem descrita em Rietveld (1978). O gerador estocástico LARS-WG utiliza uma distribuição semi-empírica (SED) que é especificada como a função de distribuição cumulativa de probabilidade (FAP) para aproximar a distribuição de probabilidade de séries de períodos secos e úmidos de precipitação diária, temperatura mínima e máxima e radiação solar diária. O número de intervalos (n) usado na SED é de 23 para variável climática (Semenov e Barrow, 2002). O LARS-WG simula séries temporais de dados meteorológicos diários com características estatísticas semelhantes a dos dados observados e podem ser usadas para: 1) gerar dados meteorológicos de séries temporais de longo prazo adequados para avaliação de risco em estudos agrícolas e hidrológicos; 2) fornecer os meios para ampliar a simulação de dados meteorológicos para locais não observados; e 3) servem como uma ferramenta computacionalmente barata para produzir cenários diários específicos de mudança de clima baseados em resultados de modelos climáticos gerais (globais) e regionais para avaliações de impacto de mudanças climáticas.

O processo de geração de dados de cenário climático diário em escala local no LARS-WG é dividido em duas etapas distintas, uma de análise/calibração e outra de geração de séries sintéticas, descritos por Semenov e Barrow (2002) e Semenov e Stratonovitch (2010). Os dados da linha de base, série temporal observada em um determinado período de tempo, são utilizados para realizar a calibração dos parâmetros a partir série observada e permitir a geração das séries de dados sintéticos usando LARS-WG 5.0. Stockle et al. (2003) e Mckague et al. (2005) recomendam o uso de pelo menos 35 anos de dados meteorológicos observados diariamente para determinar os parâmetros estatísticos. Os testes estatísticos utilizados no LARS-WG baseiam-se no pressuposto de que os dados observados e os de clima sintético são amostras aleatórias de distribuições existentes e procuram testar se a hipótese nula de que as duas distribuições são as mesmas é aceita. Os testes estatísticos utilizados neste estudo incluíram o teste de Kolmogorov-Simirnov (KS) para comparar as distribuições de probabilidade, T-test para comparar médias e F-test para comparar desvios-padrão. O LARS-WG é validado comparando as estatísticas calculadas a partir de uma série sintética de clima gerada pelo gerador de clima contra os dados meteorológicos das séries temporais observadas.

Esta versão incorpora previsões climáticas de modelos climáticos globais obtidas a partir do Centro de Distribuição de Dados do IPCC. Os autores utilizaram técnicas de downscaling estatístico para criar cenários climáticos diários em escala local. Para gerar cenários climáticos para um período no futuro com emissões específicas, os parâmetros linha base LARS-WG, calculados a partir da série de dados observada foram ajustados pelas mudanças esperadas para este período como descrito em Semenov e Stratonovitch (2010).
De acordo com Semenov e Stratonovitch (2010) e Hassan e Harun (2013), a simulação da ocorrência de precipitação é modelada como dias úmidos e secos, onde um dia úmido é definido como um dia com precipitação maior que $0,0 \mathrm{~mm}$. O comprimento da sequência de dias secos ou úmidos é escolhida aleatoriamente a partir da distribuição semi-empírica úmida ou seca para o mês em que a série se inicia. Para um dia úmido, o valor da precipitação é gerado a partir da distribuição de precipitação semi-empírica para o mês específico independente do comprimento da série úmida ou a quantidade de precipitação nos dias anteriores. Para cada variável climática $v$, um valor de uma variável climática $v i$ correspondente à probabilidade $p i$ é calculado, segundo Chen et al. (2012), como na Eq. (1):

$$
v_{i}=\min \left\{v: P\left(V_{o b s} \leq v_{i}\right) \geq p_{i}\right\}, i=0,1,2, \cdots, \mathrm{n}
$$

Em que $P($ ) denota probabilidade com base em dados observados $\{V o b s\}$. Para cada variável climática, dois valores, $p_{0}$ e $p_{n}$, são fixados como $p_{0}=0$ e $p_{n}=1$, com valores correspondentes de $v_{0}=\min \left\{V_{o b s}\right\}$ e $v_{n}=\max$ $\left\{V_{o b s}\right\}$. Para aproximar os valores extremos de uma variável climática com precisão, alguns $p_{i}$ são atribuídos perto de 0 para valores extremamente baixos da variável e perto de 1 para valores extremamente altos e os valores remanescentes de $p_{i}$ são distribuídos uniformemente na escala de probabilidade. Uma vez que a probabilidade de precipitação diária muito baixa $(<1 \mathrm{~mm})$ é tipicamente relativamente alta e baixa precipitação tem muito pouco impacto na saída de um modelo baseado em processo, dois valores de $v_{1}=0,5 \mathrm{~mm}$ e $v_{2}=1 \mathrm{~mm}$ são empregados para se aproximar a precipitação dentro do intervalo $(0,1)$ com as probabilidades correspondentes calculadas como $p_{i}=P\left(V_{o b s} \leq v_{i}\right), i=1,2$. Para representar séries secas e úmidas extremamente longas, dois valores próximos de 1 são usados nas SEDs para séries úmidas e secas, $p_{n-1}=$ 0,99 e $p_{n-1}=0,98$ (Chen et al., 2012).

\section{Material e Métodos}

\subsection{Caracterização da área de estudo}

A área de estudo compreende o município de Porto Alegre, capital do Estado do Rio Grande do Sul e, situa-se na Latitude $30^{\circ} 01 ' 58^{\prime \prime} \mathrm{S}$ e Longitude $51^{\circ} 13$ ' $48^{\prime \prime}$ W. O município apresenta área de $497 \mathrm{~km}^{2}$ (IBGE, 2010) e localiza-se a uma altitude média de 10 metros. Sua população é de 1.409.351 habitantes (IBGE, 2010). O município de Porto Alegre está localizado na sub-bacia 87 (Fig. 1) com uma área aproximada de $56.530 \mathrm{~km}^{2}$ (Marcuzzo, 2017), inserida na bacia 8 , denominada Bacia do Atlântico, trecho Sudeste, de acordo com a sub-divisão do Brasil em bacias, proposta pelo extinto Departamento Nacional de Águas e Energia Elétrica (DNAEE), em fevereiro de 1972 (Galvão e Meneses, 2005). 


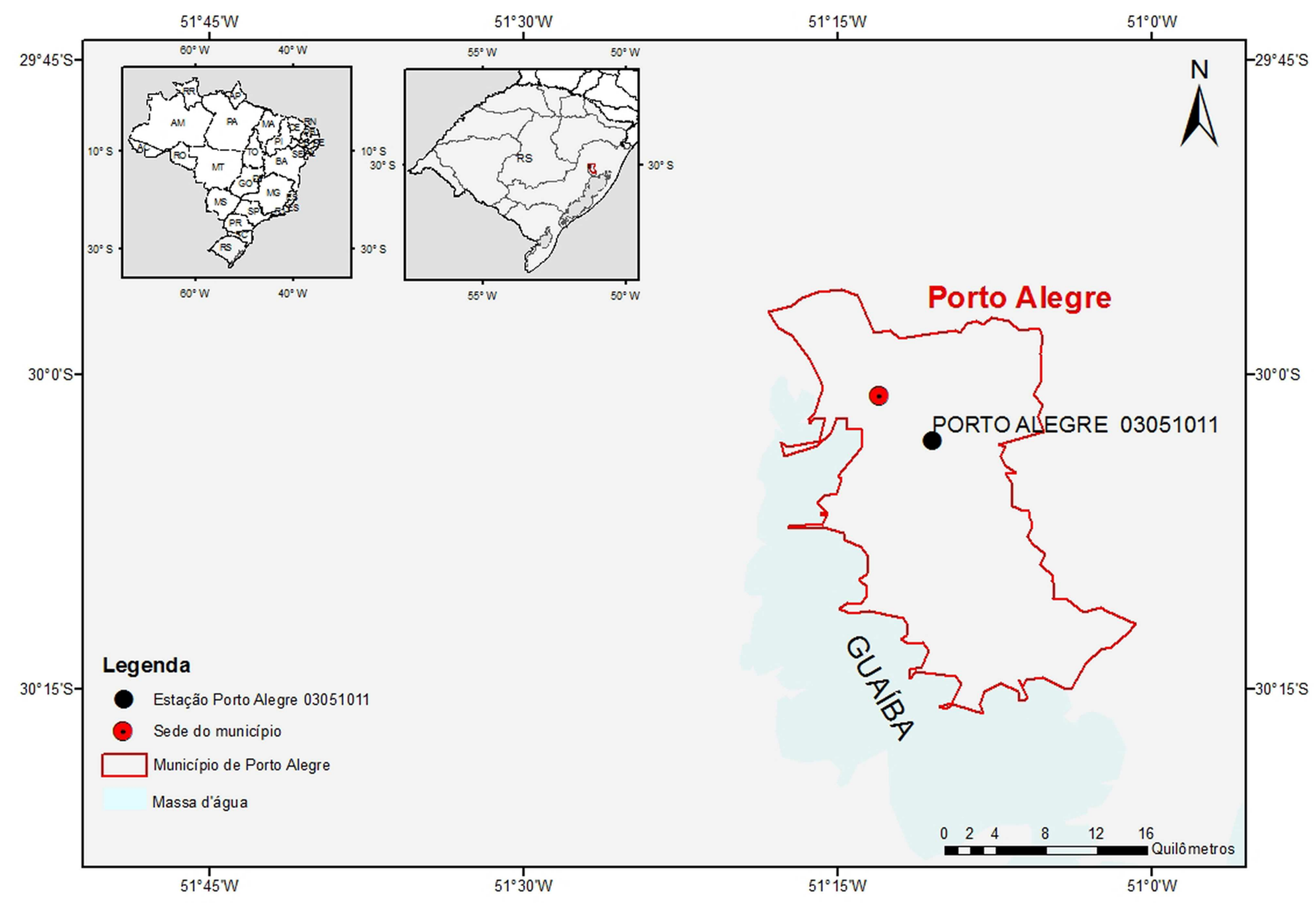

Figura 1. - Localização do Município, sub-bacia a qual pertence e estação utilizada no estudo.

A cidade de Porto Alegre tem regime de precipitação pluvial bem distribuído ao longo do ano, como pode se visualizado no hietograma (Fig. 2) com a média dos totais mensais no período de 1974 a 2014, construído com dados pluviométricos provenientes da estação com códigos 03051011 (ANA) e 83967 (INMET), operada pelo INMET (Instituto Nacional de Meteorologia). A Fig. 2 nos permite observar que mesmo com volume de precipitação bem distribuído, os meses mais representativos, com precipitação média superior a $120 \mathrm{~mm}$, vão de junho a outubro. As precipitações máximas (Fig. 3) com valores superiores a $100 \mathrm{~mm}$ ocorreram em fevereiro de 1981, maio de 2008, junho de 1982 e novembro de 2013. Salienta-se que a média do total anual da série de dados do INMET é de $1439 \mathrm{~mm}$.

\subsection{Geração da série sintética linha-base}

Utilizando a versão 5 do LARS-WG desenvolvido pelo pesquisador Mikhail Semenov, do Rothamsted Research, atualizado em agosto de 2015, antes de gerarmos as séries sintéticas para os cenários de projeção é necessária a calibração e avaliação da representatividade do modelo na geração da série sintética.
A etapa de calibração consiste em avaliar a capacidade de representação dos dados de um local no LARSWG, no caso os dados de Porto Alegre, e, é realizada através do teste de Kolmogorov Smirnov - KS (Crutcher, 1975). O teste verifica o ajuste entre a distribuição empírica, representada pelo o histograma dos dados observados de precipitações diárias, e o ajuste da distribuição dos dados sintéticos, representado pelo histograma dos dados sintéticos. Um valor de p, adotado no teste é usado para aceitar ou rejeitar a hipótese que os dois conjuntos de dados, observados e sintéticos sejam provenientes da mesma distribuição. Um p-valor muito baixo indica que deve ser rejeitada a hipótese de que as séries sejam provenientes da mesma distribuição e, por consequência, o clima simulado pela séria sintética não corresponda ao clima observado. O p-valor de 0,05 é o nível de significância comum utilizado na maioria das estatísticas, mas no LARS-WG o limite de significância de 0,01 é usado como aceitável.

A geração da série sintética teve como referência a séries de dados climatológicos, diários observados em Porto Alegre no período de 1974 a 2014, que permitiu calcular o conjunto de parâmetros referentes às distribuições de probabilidade das variáveis observadas. 
HIETOGRAMA DOS TOTAIS MENSAIS DE 1974-2014 EM PORTO ALEGRE

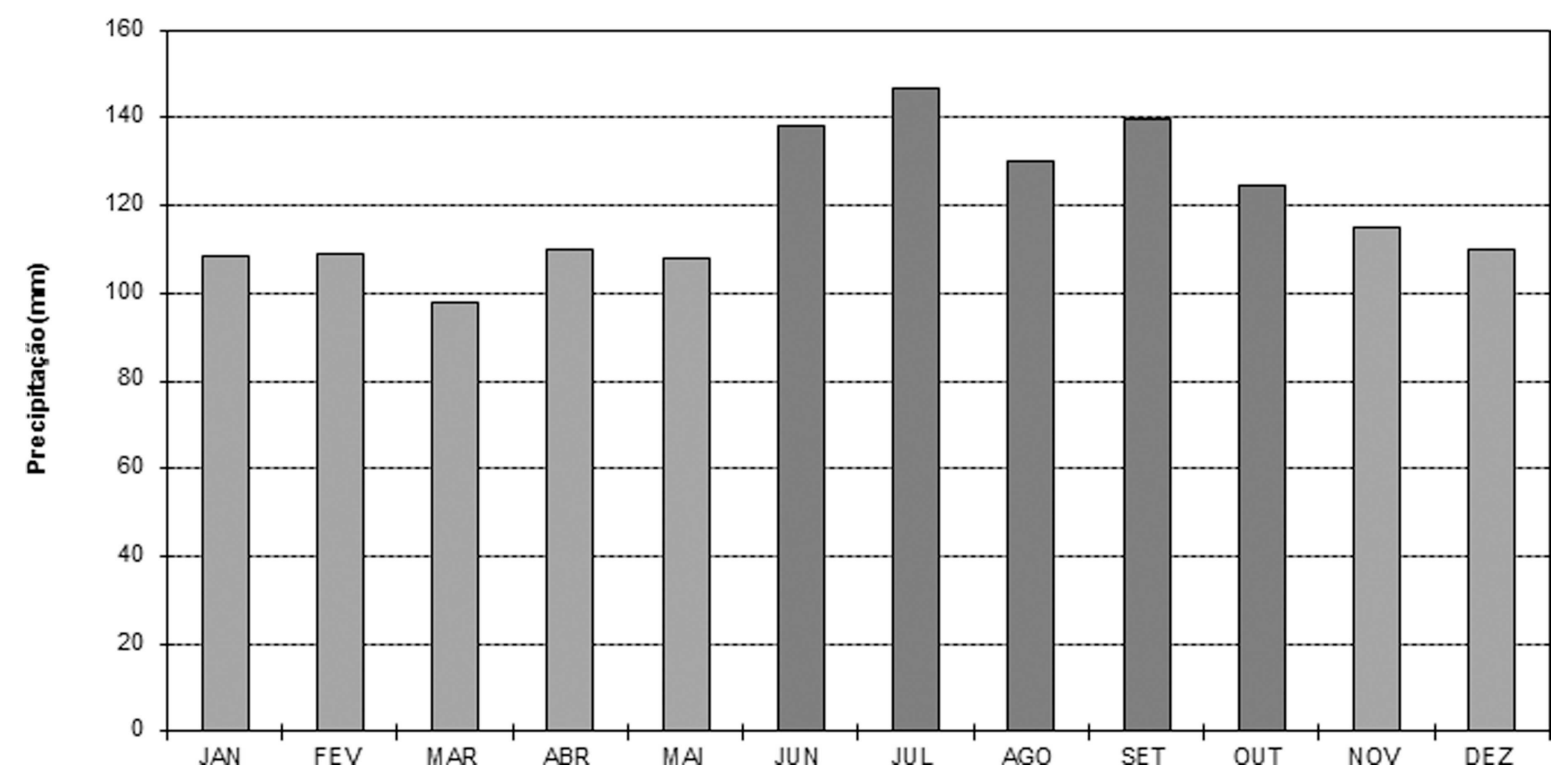

Figura 2. - Hietograma da média dos totais mensais - Porto Alegre 1974-2014.

PRECIPITAÇÕES MÁXIMAS DE 1974-2014 EM PORTO ALEGRE

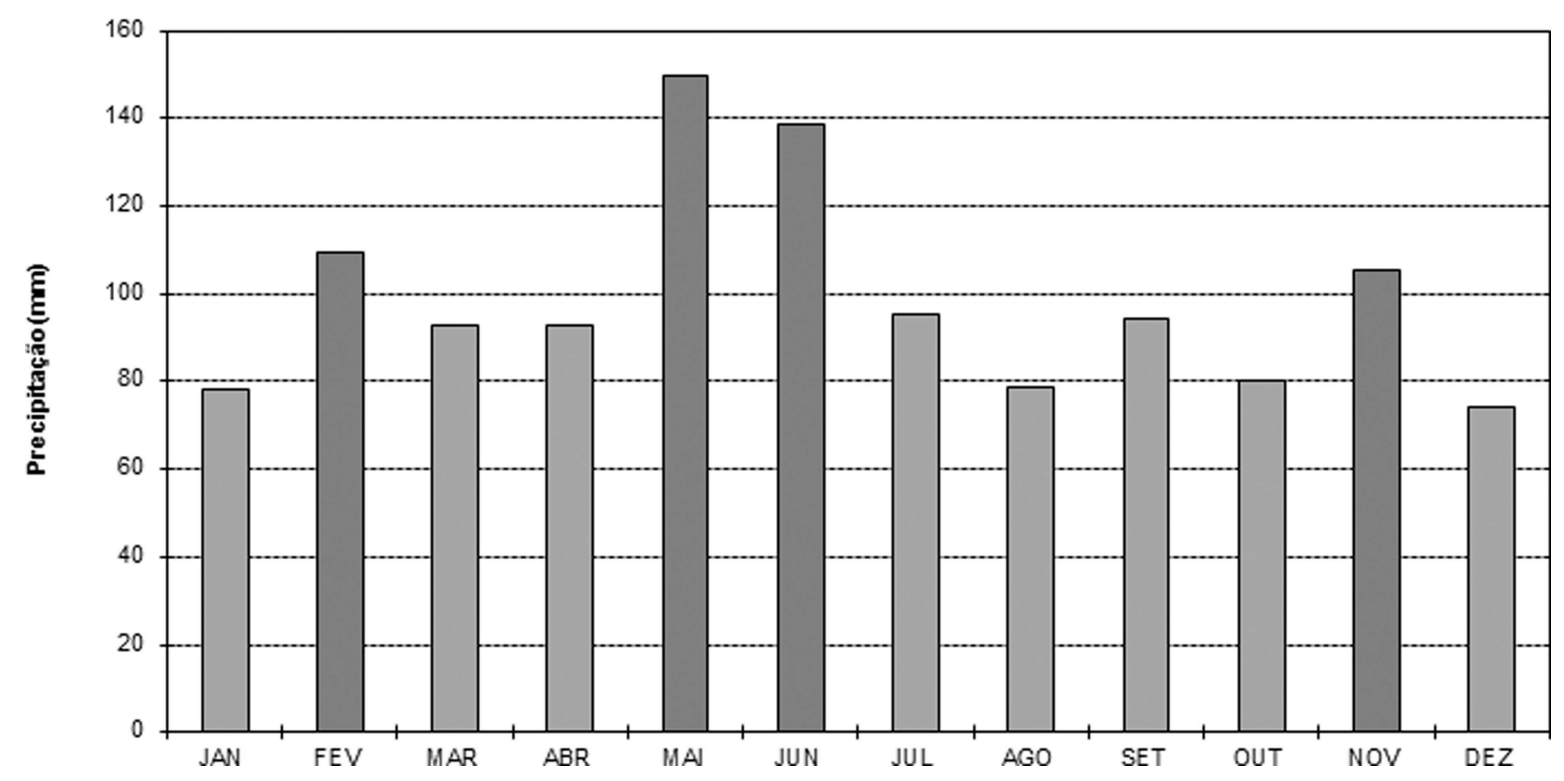

Figura 3. - Precipitações máximas ocorridas nos meses de janeiro a dezembro em Porto Alegre no período de $1974-2014$.

Com base nestes parâmetros foram geradas as séries de dados sintéticos com características estatísticas similares, de comprimento arbitrário para o clima atual denominada linha de base. Cabe salientar que as séries sintéticas para o clima atual foram geradas para as seis opções de semente.

A semente corresponde ao ponto de partida do gerador de números pseudo-aleatórios no qual se dá inicio a geração da série sintética. De acordo com Detzel (2009), uma mesma semente resultará sempre em uma mesma sequência de números pseudo-aleatórios. No LARS-WG tem-se implementada as sementes 541, 1223, 1987,2741, 3571 e 4409 , que permitem a geração de séries similares estatisticamente com a diferença em uma base dia a dia.

A quantidade e a ocorrência das variáveis climáticas no LARS-WG é dada por histogramas ajustados aos dados observados que consistem em uma distribuição semiempírica. Após o calculo da média, variância, desvios, 
coeficientes de assimetria e curtose é possível aproximar uma distribuição de probabilidade e o cálculo dos parâmetros para esta distribuição.

A distribuição semi-empírica segundo Semenov e Stratonovitch (2010) é definida como uma função de distribuição de probabilidade acumulada ou função acumulada de probabilidade construída com base em um histograma com 23 intervalos fixos.

\subsection{Desagregação da série sintética linha-base}

A série sintética de precipitação gerada no LARSWG, consiste de dados diários, entretanto o interesse neste estudo são os dados sub-diários. Assim, de posse das razões entre alturas de chuvas de diferentes durações, obtidas da IDF definida por Weschenfelder et al. (2015) que será denominada IDF atual, foi utilizada a metodologia de Pinto (2013). Através desta metodologia por meio de uma função linear transformam-se as precipitações diárias máximas anuais (Pdma) em de precipitações de 24 horas e na sequência as precipitações de 24 horas em alturas de menor duração (sub-diárias), estabelecendo assim uma curva IDF.

As etapas da metodologia aplicada de forma resumida são as seguintes:

1. Separação do máximo anual de cada ano, série de precipitações diárias máximas anuais - Pdma.

2. Realização da análise de frequência e escolha da distribuição que melhor represente a série de dados.

3. Desagregação da Pdma em diferentes durações, dados sub-diários.

Como recomendado por Naghettini e Pinto (2007), na análise de frequência foi necessário avaliar se os dados da série eram independentes, homogêneos e estacionários. Também foi necessário, identificar a presença de outliers. $\mathrm{Na}$ sequência foi estimada a distribuição empírica, os parâmetros e realizada a verificação da aderência da distribuição.

A metodologia proposta por Pinto (2013), usada neste estudo, consistiu na aplicação dos testes de Grubbs e Beck para verificação de outliers, teste de NERC para verificação da aleatoriedade, teste de Wald e Wolfowitz para verificação da independência, teste de Mann e Whitney para verificação da homogeneidade e teste de Spearman para avaliação da estacionariedade.

Para estimação da distribuição empírica primeiramente ordenou-se de forma decrescente a série e calculouse a posição de plotagem pela fórmula de Weibull, ou seja, $P(P>p)=m /(N+1)$, sendo que $m$ é número de ordem e $\mathrm{N}$ o tamanho de amostra.

$\mathrm{Na}$ sequência foram calculados os momentos- $\mathrm{L}$ amostrais das séries por duração e estimados os parâmetros das distribuições teóricas candidatas a representar a série.

$\mathrm{Na}$ escolha das distribuições teóricas que melhor representa a distribuição empírica, além da análise visual no gráfico de probabilidade de Gumbel foi realizado o teste de aderência de KS.

Após escolher a distribuição teórica foram definidos os quantis ou valores associados a uma probabilidade de excedência para diferentes tempos de retorno. O maior tempo de retorno utilizado para estimar o quantil no caso dos dados sintéticos da linha de base foi de 100 anos.

Concluída a análise de frequência da série sintética diária, foi feita a desagregação propriamente dita, que visa à transformação dos dados diários em precipitações com duração de 24 horas e sub-diárias. Por meio de uma função linear, transformam-se as precipitações diárias em chuvas de 24 horas utilizando a relação $24 \mathrm{~h} / 1$ dia de 1,14 , valor médio recomendado pela CETESB - Companhia Ambiental do Estado de São Paulo. Na sequência, a desagregação da precipitação de 24 horas em alturas de chuva de menor duração utiliza as razões entre as alturas de precipitação de diferentes durações das IDF definidas por Weschenfelder et al. (2015) apresentada na Tabela 1.

As curvas IDF ajustadas neste estudo foram do tipo sugerido por Bertoni e Tucci (2004) na qual o ajuste de uma distribuição estatística aos maiores valores da série (Pdma), pode ser expresso na forma gráfica e por equações genéricas como na forma da Eq. (2):

$$
i=\frac{a * T r^{b}}{(t+c)^{d}}
$$

Na Eq. (2) $i$ é a intensidade da precipitação geralmente expressa em $\mathrm{mm} / \mathrm{h}, T r$ é o tempo de retorno dado em anos, $t$ é a duração da precipitação em minutos e $a, b, c, d$ correspondem aos parâmetros da equação. Estes parâmetros serão determinados para linha de base e para cada projeção na etapa de simulação dos cenários de projeção.

De posse dos quantis desagregados a partir das séries sintéticas efetuou-se a comparação com os quantis calculados para as diferentes durações da IDF atual. Para tanto foi efetuada a análise dos desvios percentuais, desvios percentuais médios, e desvios percentuais absoluto médio. $O$ desvio percentual expressa a diferença entre o valor da IDF atual e o valor da IDF da linha de base em percentagem, obtido para as diferentes sementes para cada uma das durações e diferentes tempos de retorno. A equação para o cálculo do desvio percentual é bem simples e pode ser expressa por $((V f-V i)) / V i * 100)$. Sendo que $V f$ ou valor final é o valor do quantil da linha de base e $V i$ ou valor inicial o valor do quantil da IDF atual. O desvio absoluto percentual é o desvio percentual em módulo.

Tabela 1 - Razões entre as alturas de precipitação de diferentes durações definidas para Porto Alegre. Fonte: Weschenfelder et al. (2015).

\begin{tabular}{ccccccccc}
\hline $14 \mathrm{~h} /$ & $8 \mathrm{~h} /$ & $4 \mathrm{~h} /$ & $2 \mathrm{~h} /$ & $1 \mathrm{~h} /$ & $45 \mathrm{~min} /$ & $30 \mathrm{~min} /$ & $15 \mathrm{~min} /$ & $5 \mathrm{~min} /$ \\
$24 \mathrm{~h}$ & $24 \mathrm{~h}$ & $24 \mathrm{~h}$ & $24 \mathrm{~h}$ & $24 \mathrm{~h}$ & $1 \mathrm{~h}$ & $1 \mathrm{~h}$ & $1 \mathrm{~h}$ & $1 \mathrm{~h}$ \\
\hline 0,85 & 0,67 & 0,56 & 0,51 & 0,47 & 0,91 & 0,77 & 0,52 & 0,26 \\
\hline
\end{tabular}




\subsection{Simulação dos cenários projetados}

Neste trabalho foram geradas séries sintéticas de precipitação diária baseadas em três cenários de emissões do quarto relatório do Painel Intergovernamental de mudanças climática, A1B, A2 e B1, considerando dois períodos de projeção 2046 a 2065 e de 2080 a 2099, e seis modelos de circulação global (MCG). (IPCC-AR4). As simulações resultaram em 216 trajetórias de precipitações associadas as emissões previstas de concentrações de $\mathrm{CO}_{2}$ apresentadas na Tabela 2 e simuladas nos seis MCG listados na Tabela 3.

Os cenários indicam o que pode ocorrer no futuro em diferentes condições de avanço tecnológico e social, aumento demográfico e emissões de gases de efeito estufa (GEE). Na Tabela 2 o cenário mais pessimista é o cenário A2 com manutenção dos padrões de emissão dos GEE observados nas últimas décadas, o mais otimista o cenário B1 com estabilização das emissões e o A1B corresponde a um cenário intermediário.

Segundo Goosse et al. (2010), os cenários fornecem estimativas de emissões futuras do principal gás de efeito estufa o $\mathrm{CO}_{2}$ mas também de outros gases de efeito estufa como $\mathrm{N}_{2} \mathrm{O}$ e $\mathrm{CH}_{4}$ e de emissões de dióxido de enxofre $\left(\mathrm{SO}_{2}\right)$ que esta relacionada a produção de aerossóis de sulfato na atmosfera.

Os modelos selecionados são listados na Tabela 3, e estão implementados no LARS-WG, foram escolhidos por apresentarem os mesmos cenários de projeção, A1B, B1, e A2 o que permite analise comparativa entre os resultados projetados, NCCCSM, MPEH5, IPCM4, GFCM21, HADCM3, INCM3.

As informações de alterações climáticas previstas para determinado cenário, modelo e período, incorporadas

Tabela 2 - Cenários de emissões do quarto relatório do Painel Intergovernamental de mudanças climática ( IPCC-AR4). Fonte: Modificado de Semenov (2010).

\begin{tabular}{|c|c|c|}
\hline Cenário & Concentração $\mathrm{CO}_{2}(\mathrm{ppm})$ 2046-2065 & $2081-2100$ \\
\hline B1 & 492 & 538 \\
\hline A1B & 546 & 174 \\
\hline A2 & 545 & 754 \\
\hline
\end{tabular}

Tabela 3 - Modelos selecionados nas projeções utilizadas neste estudo.

\begin{tabular}{lcc}
\hline $\begin{array}{l}\text { Modelo de Circula- } \\
\text { ção Geral }\end{array}$ & $\begin{array}{c}\text { Nome no LARS- } \\
\text { WG }\end{array}$ & $\begin{array}{c}\text { Resolução espacial (latitude } \\
\text { X longitude) }\end{array}$ \\
\hline NCCCSM & CCSM3 & $1,4 \times 1,4$ \\
MPEH5 & ECHAM5 & $1,9 \times 1,9$ \\
IPCM4 & IPSL-CM4 & $2,5 \times 3,75$ \\
GFCM21 & GFDL-CM2.1 & $2,0 \times 2,5$ \\
HADCM3 & UMKO- & $2,5 \times 3,75$ \\
& HADCM3 & \\
INCM3 & INM-CM3 & $4,0 \times 5,0$ \\
\hline
\end{tabular}

no LARS-WG, consistem em um cenário perturbado que utiliza o arquivo de parâmetros dos dados observados, armazenado na etapa de calibração do modelo.

A geração das séries sintéticas para trajetórias futuras considera um cenário que corresponde ao clima atual, representado pela linha de base e um cenário perturbado num período futuro, para determinado modelo. As mudanças previstas no cenário perturbado levam em conta as emissões de gases de efeito estufa e sulfato e as mudanças relativas na precipitação média e na duração do período seco e chuvoso entre outras variáveis como temperatura e radiação.

Foram geradas séries sintéticas de 300 anos de dados, pois esta duração seria adequada conforme explicitado por Semenov e Barrow (2002). Semenov (2010) também coloca que para cada MCG e combinações das emissões dos cenários em um período de tempo futuro, 300 anos de dados diários são gerados de acordo com cenários climáticos específicos de um local e usados pelo modelo em um processo para calcular as estatísticas de impacto selecionadas.

\subsection{Desagregação das séries sintéticas geradas por cenário de projeção}

Na seleção dos máximos diários anuais sintéticos, a serem usados na geração das curvas IDFs pelo método da desagregação, para cada cenário, período e modelo foram selecionados apenas os últimos 40 anos da série. Desta forma, permitiu-se um bom afastamento da primeira geração ou primeiros sorteios realizados pelo modelo, o chamado aquecimento. Assim, será garantido que estes eventos são totalmente desvinculados dos primeiros valores gerados da série sintética, e, também se tem facilitada a análise comparativa com a IDF atual de Porto Alegre que foi construída com uma série histórica de 40 anos de observações.

O procedimento para realizar a desagregação dos dados diários sintéticos gerados, em sub-diários, para um determinado cenário de alteração climática seguiu exatamente os mesmos passos da desagregação dos dados sintéticos gerados na obtenção das relações IDF para linha de base. A partir da série sintética obtida por projeção, efetuou se a separação dos máximos na geração da Pdma, análise frequência, seleção da distribuição e desagregação dos dados diários. Também, foram realizados os testes estatísticos, para avaliar a aleatoriedade, independência, homogeneidade e estacionáriedade e aderência da distribuição estatística aos dados empíricos.

Após ter sido estimada a distribuição empírica, os parâmetros e efetuada a verificação da aderência de uma distribuição (exponencial) foi realizada a análise comparativa dos quantis calculados.

Os quantis nas diferentes durações da IDF atual foram comparados com o quantis obtidos para as IDFs projetadas para os cenários A1B, A2 e B1 para cada um 
dos modelos, considerando o período de projeção de 20462065 e de 2080-2099 para durações de cinco minutos a 24 horas.

A análise comparativa dos resultados obtidos para cada um dos três cenários e períodos de projeção um e dois foi comparada com IDF atual e efetuada pelo desvio percentual (DP), desvio percentual médio (DPM).

O desvio percentual é a diferença em percentual, para diferentes tempos de retorno, entre o valor obtido para cada uma das durações da IDF atual e o valor obtido para IDF projetada. O desvio percentual médio é diferença entre o valor que corresponde à média dos valores que foram comparados com a IDF atual. A média pode ser a media dos valores obtido para as diferentes sementes por modelo, cenário e projeção ou ainda a média destes valores obtidos para cada modelo para cada cenário e período de projeção.

$\mathrm{Na}$ interpretação do conjunto de dados que corresponde aos valores de desvio dos quantis obtidos num cenário projetado em relação aos quantis atuais foram utilizados gráficos do tipo box-plot. O gráfico do tipo boxplot fornece um resumo visual da estatística dos dados, como mediana, intervalo interquartil, assimetria e presença de valores atípicos. Segundo Helsel e Hirsch (2002), box-plots são muitas vezes colocados lado a lado para visualmente comparar e contrastar grupos de dados.

Neste estudo o diagrama box-plot gerado no software $\mathrm{R}$ consistiu em um retângulo na vertical, cujo topo corresponde ao terceiro quartil e a base ao primeiro quartil. A mediana fica em seu interior no sentido transversal. Conforme exemplificado em Naghettini e Pinto (2007) a identificação de valores atípicos os denominados outliers superior e inferior é determinada da seguinte forma: traçase uma linha até o ponto que não exceda $\left(\mathrm{Q}_{3}+1,5 \mathrm{AIQ}\right)$, considerado o limite superior, onde $\mathrm{Q}_{3}$ corresponde ao terceiro quartil e AIQ corresponde ao intervalo interquartil $\left(Q_{3}-Q_{1}\right)$. E, de modo análogo traça-se outra linha a partir do lado inferior do retângulo até o limite inferior dado por $\left(\mathrm{Q}_{1}-1,5 \mathrm{AIQ}\right)$, com $\mathrm{Q}_{1}$ correspondendo ao primeiro quartil e AIQ ao intervalo interquartil. As observações acima ou abaixo destes limites são consideradas os outliers. E, de modo análogo traça-se outra linha a partir do lado inferior do retângulo até o limite inferior dado por $\left(\mathrm{Q}_{1}-1,5 \mathrm{AIQ}\right)$, com $\mathrm{Q}_{1}$ correspondendo ao primeiro quartil e AIQ ao intervalo interquartil. As observações acima ou abaixo destes limites são consideradas os outliers.

\section{Resultados e Discussão}

\subsection{Calibração}

Os resultados obtidos após a calibração, que consistiu na realização de testes que avaliam a igualdade das distribuições estatísticas entre os dados observados e os dados sintéticos, são apresentados nas Tabelas 4 e 5.
Tabela 4 - Teste de Kolmogov-Smirnov para verificar o ajuste da série de precipitação diária ao período seco e chuvoso nos trimestres que correspondem ao verão (DJD), outono (MAM), inverno (JJA) e primavera (SON).

\begin{tabular}{lccc}
\hline Trimestre & Teste & P. seco & P. chuvoso \\
\hline DJF & KS & 0,044 & 0,055 \\
& p-valor & 1,000 & 1,000 \\
\multirow{2}{*}{ MAM } & KS & 0,036 & 0,054 \\
& p-valor & 1,000 & 1,000 \\
JJA & KS & 0,077 & 0,137 \\
& p-valor & 1,000 & 0,972 \\
\multirow{2}{*}{ SON } & KS & 0,038 & 0,055 \\
& p-valor & 1,000 & 1,000 \\
\hline
\end{tabular}

Tabela 5 - Teste de Kolmogorov-Smirnov para verificar o ajuste da distribuição da série de precipitação diária aos dados mensais.

\begin{tabular}{|c|c|c|}
\hline Mês & Teste & Precipitação diária \\
\hline \multirow[t]{2}{*}{ Janeiro } & KS & 0,053 \\
\hline & p-valor & 1,000 \\
\hline \multirow[t]{2}{*}{ Fevereiro } & KS & 0,048 \\
\hline & p-valor & 1,000 \\
\hline \multirow[t]{2}{*}{ Março } & $\mathrm{KS}$ & 0,053 \\
\hline & p-valor & 1,000 \\
\hline \multirow[t]{2}{*}{ Abril } & KS & 0,007 \\
\hline & $\mathrm{p}$-valor & 1,000 \\
\hline \multirow[t]{2}{*}{ Maio } & KS & 0,043 \\
\hline & p-valor & 1,000 \\
\hline \multirow[t]{2}{*}{ Junho } & KS & 0,179 \\
\hline & p-valor & 0,816 \\
\hline \multirow[t]{2}{*}{ Julho } & KS & 0,090 \\
\hline & $\mathrm{p}$-valor & 1,000 \\
\hline \multirow[t]{2}{*}{ Agosto } & KS & 0,101 \\
\hline & p-valor & 0,999 \\
\hline \multirow[t]{2}{*}{ Setembro } & $\mathrm{KS}$ & 0,131 \\
\hline & p-valor & 0,982 \\
\hline \multirow[t]{2}{*}{ Outubro } & KS & 0,047 \\
\hline & $\mathrm{p}$-valor & 1,000 \\
\hline \multirow[t]{2}{*}{ Novembro } & $\mathrm{KS}$ & 0,104 \\
\hline & p-valor & 0,999 \\
\hline \multirow[t]{2}{*}{ Dezembro } & KS & 0,053 \\
\hline & $\mathrm{p}$-valor & 1,000 \\
\hline
\end{tabular}

Os resultados encontrados apontam para p-valores maiores que 0,8 e, assim, muito próximos de 1 o que permitem a aprovação na estatística de teste de KolmogorovSmirnov. Assim, o desempenho do LARS-WG tanto na simulação da distribuição sazonal de períodos chuvosos e secos quanto na simulação da distribuição das precipitações diárias em todos os meses indicam que ele pode ser utilizado na geração das séries sintéticas de precipitação para Porto Alegre. 
Na sequência nas Tabelas 6 e 7, são apresentadas os ajustes das distribuições estatísticas as séries de precipitações sintéticas gerada pelo LARS-WG com cada umas das seis opções de semente disponíveis, a saber, 541, $1223,1987,2741,3571$ e 4409.

Tabela 6 - Resultados do ajuste dos dados trimestrais ao período chuvoso, obtidos para cada uma das sementes disponíveis no LARS-WG.

\begin{tabular}{lccccccc}
\hline & & \multicolumn{6}{c}{ Período chuvoso } \\
\cline { 3 - 8 } Trimestre & Teste & 541 & 1223 & 1987 & 2741 & 3541 & 4409 \\
\hline DJF & KS & 0,055 & 0,273 & 0,055 & 0,277 & 0,275 & 0,271 \\
& p-valor & 1,000 & 0,307 & 1,000 & 0,290 & 0,298 & 0,315 \\
\multirow{2}{*}{ MAM } & KS & 0,054 & 0,059 & 0,048 & 0,055 & 0,038 & 0,044 \\
& p-valor & 1,000 & 1,000 & 1,000 & 1,000 & 1,000 & 1,000 \\
\multirow{2}{*}{ JJA } & KS & 0,140 & 0,116 & 0,140 & 0,145 & 0,141 & 0,142 \\
& p-valor & 0,969 & 0,996 & 0,966 & 0,995 & 0,964 & 0,962 \\
\hline
\end{tabular}

DJF corresponde ao trimestre entre dezembro e fevereiro, MAM ao trimestre entre março e abril, JJA ao trimestre entre junho e agosto e SON ao trimestre entre setembro e novembro.

Tabela 7 - Resultados do ajuste dos dados diários obtidos para cada uma das sementes.

\begin{tabular}{|c|c|c|c|c|c|c|c|}
\hline \multirow[b]{2}{*}{ Mês } & \multirow[b]{2}{*}{ Teste } & \multicolumn{6}{|c|}{ Precipitação diária } \\
\hline & & 541 & 1223 & 1987 & 2741 & 3571 & 4409 \\
\hline \multirow[t]{2}{*}{ JAN } & KS & 0,048 & 0,049 & 0,049 & 0,047 & 0,047 & 0,049 \\
\hline & p-valor & 1,000 & 1,000 & 1,000 & 1,000 & 1,000 & 1,000 \\
\hline \multirow[t]{2}{*}{ FEV } & KS & 0,013 & 0,025 & 0,060 & 0,030 & 0,081 & 0,048 \\
\hline & p-valor & 1,000 & 1,000 & 1,000 & 1,000 & 1,000 & 1,000 \\
\hline \multirow[t]{2}{*}{ MAR } & $\mathrm{KS}$ & 0,058 & 0,054 & 0,052 & 0,068 & 0,057 & 0,049 \\
\hline & p-valor & 1,000 & 1,000 & 1,000 & 1,000 & 1,000 & 1,000 \\
\hline \multirow[t]{2}{*}{$\mathrm{ABR}$} & KS & 0,046 & 0,061 & 0,006 & 0,050 & 0,046 & 0,046 \\
\hline & p-valor & 1,000 & 1,000 & 1,000 & 1,000 & 1,000 & 1,000 \\
\hline \multirow[t]{2}{*}{ MAI } & KS & 0,042 & 0,042 & 0,048 & 0,046 & 0,042 & 0,045 \\
\hline & p-valor & 1,000 & 1,000 & 1,000 & 1,000 & 1,000 & 1,000 \\
\hline \multirow[t]{2}{*}{ JUN } & KS & 0,179 & 0,198 & 0,183 & 0,181 & 0,193 & 0,182 \\
\hline & p-valor & 0,816 & 0,708 & 0,708 & 0,805 & 0,738 & 0,799 \\
\hline \multirow[t]{2}{*}{ JUL } & KS & 0,051 & 0,071 & 0,088 & 0,075 & 0,079 & 0,066 \\
\hline & p-valor & 1,000 & 1,000 & 1,000 & 1,000 & 1,000 & 1,000 \\
\hline \multirow[t]{2}{*}{ AGO } & KS & 0,096 & 0,100 & 0,105 & 0,104 & 0,125 & 0,116 \\
\hline & p-valor & 0,999 & 0,999 & 0,999 & 0,909 & 0,989 & 0,996 \\
\hline \multirow[t]{2}{*}{ SET } & $\mathrm{KS}$ & 0,124 & 0,123 & 0,126 & 0,132 & 0,115 & 0,119 \\
\hline & p-valor & 0,990 & 0,991 & 0,991 & 0,981 & 0,996 & 0,994 \\
\hline \multirow[t]{2}{*}{ OUT } & $\mathrm{KS}$ & 0,048 & 0,048 & 0,047 & 0,048 & 0,049 & 0,048 \\
\hline & $\mathrm{p}$-valor & 1,000 & 1,000 & 1,000 & 1,000 & 1,000 & 1,000 \\
\hline \multirow[t]{2}{*}{ NOV } & $\mathrm{KS}$ & 0,108 & 0,096 & 0,103 & 0,106 & 0,094 & 0,091 \\
\hline & p-valor & 0,999 & 0,999 & 0,999 & 0,999 & 0,999 & 1,000 \\
\hline \multirow[t]{2}{*}{ DEZ } & KS & 0,043 & 0,011 & 0,025 & 0,050 & 0,049 & 0,050 \\
\hline & p-valor & 1,000 & 1,000 & 1,000 & 1,000 & 1,000 & 1,000 \\
\hline
\end{tabular}

Os resultados obtidos para as sementes, foram de ajuste perfeito com p-valor igual ou muito próximo a um (1) a simplesmente bom com p-valor na faixa de 0,3 .

\subsection{IDFs projetadas $x$ IDF atual}

Os valores dos quantis obtidos após a desagregação da série sintética gerada por projeção pelo gerador estocástico reflete a variabilidade esperada para um clima futuro.

A partir das séries sintéticas geradas para trajetórias futuras com a introdução de alterações previstas pelos modelos do IPCC-AR4 disponíveis no LARS-WG, foi definida a série de precipitações diárias máxima anual (Pdma) com 40 anos.

Ao comparar os quantis da IDF atual com os quantis das IDFs projetadas, os desvios percentuais (Fig. 4), e percentuais médios (Fig. 5), apresentaram maior variabilidade para o segundo período projetado, em especial para o cenário $\mathrm{A} 2$, o mais pessimista.

No primeiro período de projeção (2046-2065) os desvios percentuais e percentuais médios para $50 \%$ dos quantis (mediana) ficaram abaixo de $15 \%$ de DP e $12 \%$ de DPM no cenário mais otimista $\mathrm{B} 1$, e abaixo de $15 \%$ de DP e DPM para os cenários A1B e A2. O que indica a possibilidade de sobrecarga no sistema de drenagem e necessidade de adequação de obras hidráulicas no futuro.

Os desvios percentuais e percentuais médios no segundo período de projeção (2080-2099), para 50\% dos quantis (mediana), ficaram abaixo de $17 \%$, considerando os cenários A1B e $\mathrm{B} 1$. E, para o cenário $\mathrm{A} 2$, os desvios ficaram abaixo de $14 \%$, o que indica a possibilidade de sobrecarga maior no sistema de drenagem, mas por se referir a um período de projeção mais distante, é maior a incerteza dos modelos.

Os resultados encontrados para Porto Alegre corroboram os resultados encontrados por Schardong e Simonovic (2013) para cidade de São Paulo. Os resultados encontrados pelos autores, para as IDFs ajustadas, aos dados sintéticos gerados, utilizando o um dos modelos do Canadian Centre for Climate Modelling and Analysis CCCma, dois cenários de emissão A2 e B1 e dois períodos de projeção quando comparados com a IDF ajustada aos dados históricos indicam um aumento entre 4 e $26 \%$ na precipitação.

$\mathrm{Na}$ sequência são apresentados a média do desvio percentual médio dos quantis, obtidos após as precipitações diárias desagregadas, em cada um dos cenários e período de projeção, considerando os diferentes modelos utilizados na geração dos dados. Nas Tabelas de 8 a 10 são apresentadas as estatísticas.

Considerando o cenário intermediário A1B e o primeiro período de projeção na Tabela 8 , os modelos HADCM3, IPCM4 e NCCCSM apresentaram em média resultados semelhantes com desvios na faixa de 12 a $16 \%$. O GFCM21 apresentou desvios menores que $8 \%$ e o 

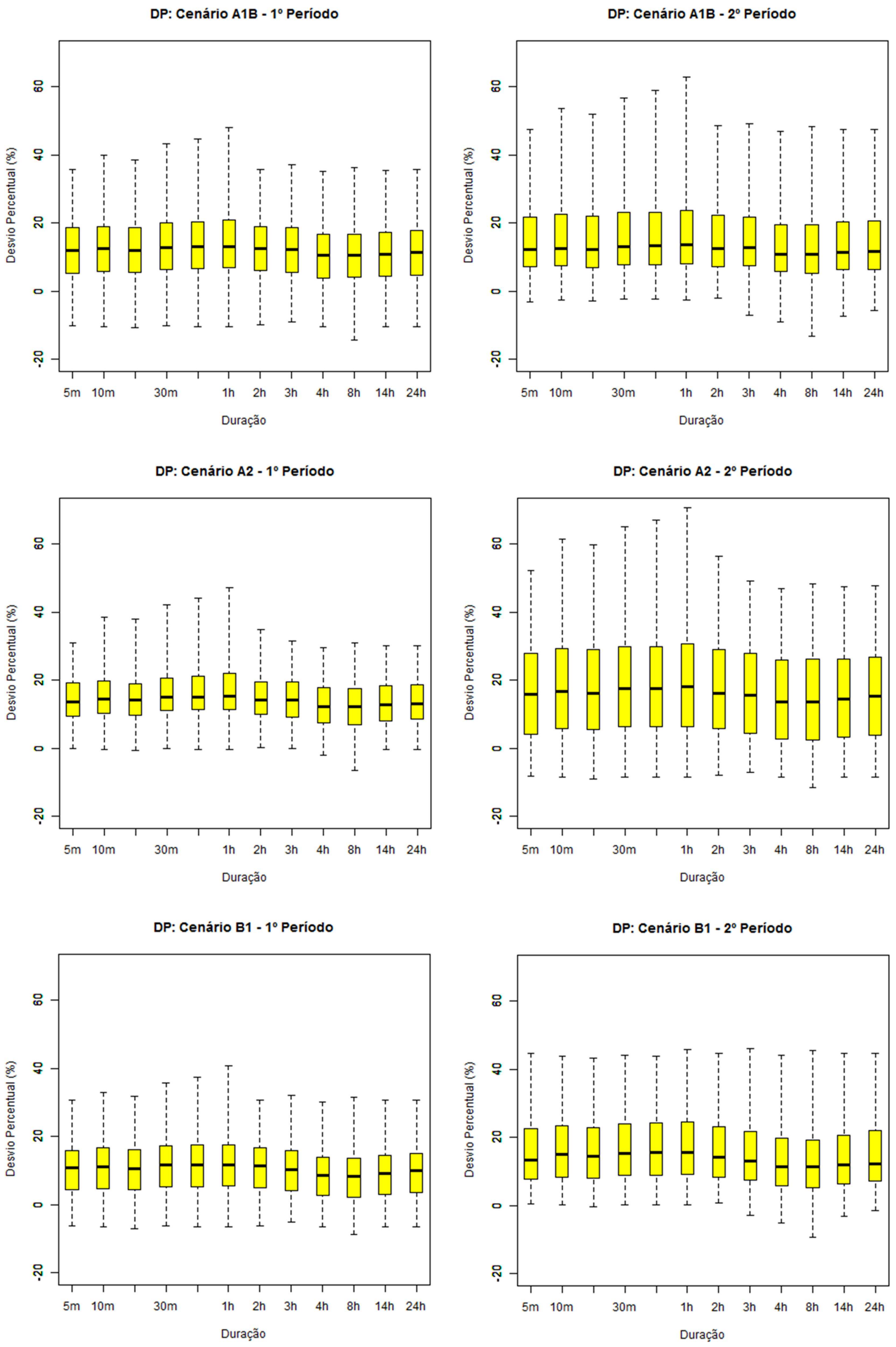

Figura 4. - Desvio percentual por cenário e período de projeção em relação aos quantis da IDF atual. 

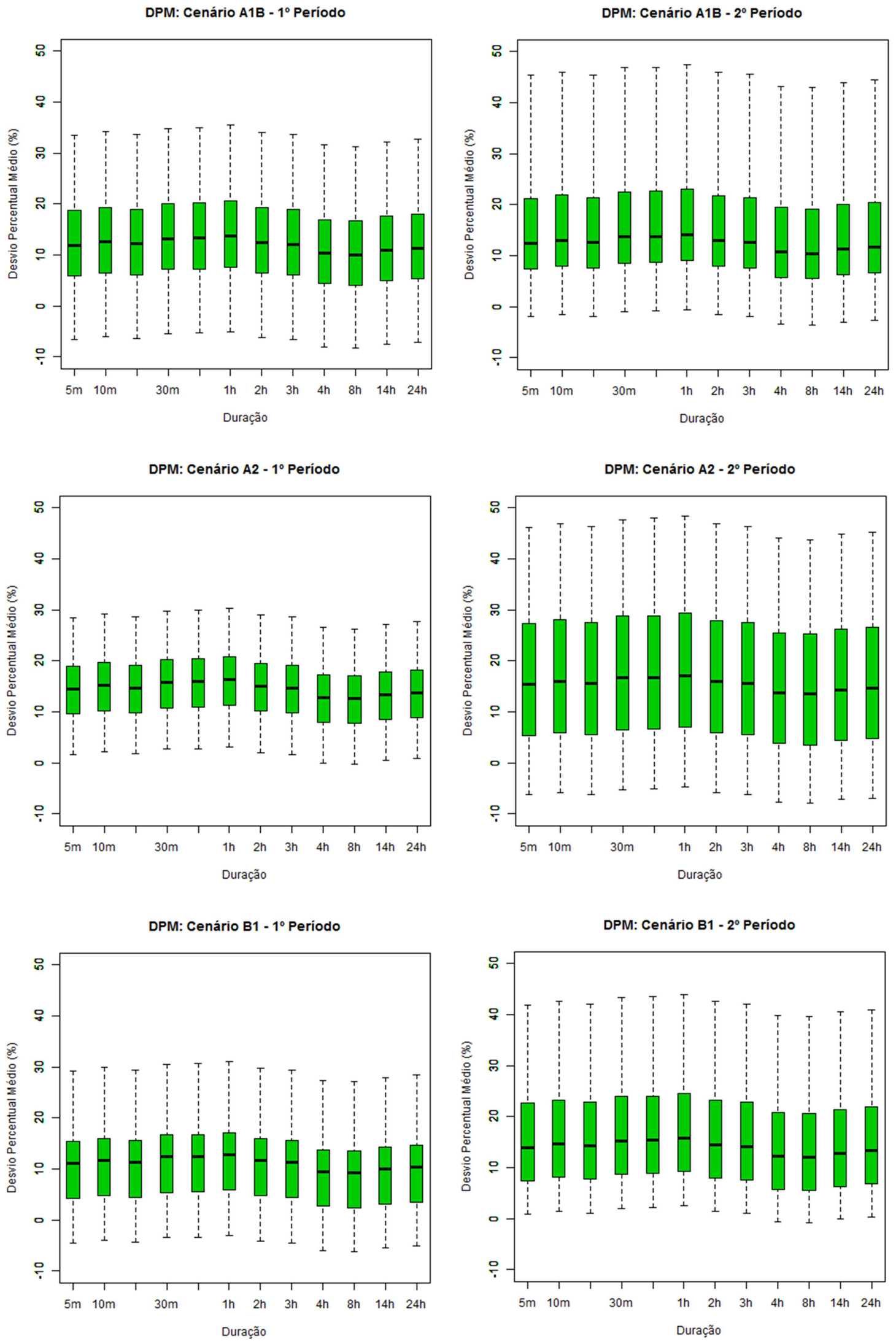

Figura 5. - Desvio percentual médio por cenário e período de projeção em relação aos quantis da IDF atual. 
Tabela 8 - Média do desvio percentual médio cenário A1B por período nas diferentes durações.

\begin{tabular}{|c|c|c|c|c|c|c|c|c|c|c|c|c|}
\hline \multicolumn{13}{|c|}{ Período 2046-2065 } \\
\hline & $5 \mathrm{~m}$ & $10 \mathrm{~m}$ & $15 \mathrm{~m}$ & $30 \mathrm{~m}$ & $45 \mathrm{~m}$ & $1 \mathrm{~h}$ & $2 \mathrm{~h}$ & $3 \mathrm{~h}$ & $4 \mathrm{~h}$ & $8 \mathrm{~h}$ & $14 \mathrm{~h}$ & $24 \mathrm{~h}$ \\
\hline GFCM21 & 7 & 7 & 7 & 8 & 8 & 8 & 7 & 7 & 5 & 5 & 5 & 6 \\
\hline HADCM3 & 14 & 15 & 15 & 16 & 16 & 16 & 15 & 15 & 13 & 12 & 13 & 14 \\
\hline INCM3 & 1 & 2 & 1 & 2 & 3 & 3 & 2 & 1 & 0 & 0 & 0 & 1 \\
\hline IPCM4 & 15 & 15 & 15 & 16 & 16 & 16 & 15 & 15 & 13 & 13 & 14 & 14 \\
\hline MPEH5 & 27 & 28 & 27 & 28 & 28 & 29 & 27 & 27 & 25 & 25 & 26 & 26 \\
\hline NCCCSM & 12 & 13 & 13 & 14 & 14 & 14 & 13 & 13 & 11 & 10 & 11 & 12 \\
\hline \multicolumn{13}{|c|}{ Períodon 2080-2099 } \\
\hline & $5 \mathrm{~m}$ & $10 \mathrm{~m}$ & $15 \mathrm{~m}$ & $30 \mathrm{~m}$ & $45 \mathrm{~m}$ & $1 \mathrm{~h}$ & $2 \mathrm{~h}$ & $3 \mathrm{~h}$ & $4 \mathrm{~h}$ & $8 \mathrm{~h}$ & $14 \mathrm{~h}$ & $24 \mathrm{~h}$ \\
\hline GFCM21 & 1 & 1 & 1 & 2 & 2 & 2 & 1 & 1 & -1 & -1 & 0 & 0 \\
\hline HADCM3 & 14 & 15 & 14 & 15 & 16 & 16 & 15 & 14 & 12 & 12 & 13 & 13 \\
\hline INCM3 & 9 & 10 & 9 & 10 & 10 & 11 & 10 & 9 & 7 & 7 & 8 & 8 \\
\hline IPCM4 & 12 & 13 & 12 & 13 & 13 & 14 & 13 & 12 & 10 & 10 & 11 & 11 \\
\hline MPEH5 & 38 & 39 & 39 & 40 & 40 & 40 & 39 & 39 & 36 & 36 & 37 & 38 \\
\hline NCCCSM & 19 & 20 & 19 & 20 & 20 & 21 & 19 & 19 & 17 & 17 & 18 & 18 \\
\hline
\end{tabular}

Tabela 9 - Média do desvio percentual médio cenário A2 por período nas diferentes durações.

\begin{tabular}{|c|c|c|c|c|c|c|c|c|c|c|c|c|}
\hline \multicolumn{13}{|c|}{ Período 2046-2065 } \\
\hline & $5 \mathrm{~m}$ & $10 \mathrm{~m}$ & $15 \mathrm{~m}$ & $30 \mathrm{~m}$ & $45 \mathrm{~m}$ & $1 \mathrm{~h}$ & $2 \mathrm{~h}$ & $3 \mathrm{~h}$ & $4 \mathrm{~h}$ & $8 \mathrm{~h}$ & $14 \mathrm{~h}$ & $24 \mathrm{~h}$ \\
\hline GFCM21 & 12 & 13 & 12 & 13 & 13 & 14 & 13 & 12 & 10 & 10 & 11 & 11 \\
\hline HADCM3 & 10 & 11 & 10 & 11 & 12 & 12 & 11 & 10 & 9 & 8 & 9 & 10 \\
\hline INCM3 & 11 & 12 & 12 & 13 & 13 & 13 & 12 & 12 & 10 & 10 & 10 & 11 \\
\hline IPCM4 & 13 & 13 & 13 & 14 & 14 & 14 & 13 & 13 & 13 & 11 & 12 & 12 \\
\hline MPEH5 & 22 & 22 & 22 & 23 & 23 & 24 & 22 & 22 & 20 & 20 & 20 & 21 \\
\hline NCCCSM & 19 & 20 & 19 & 21 & 21 & 21 & 20 & 19 & 18 & 17 & 18 & 19 \\
\hline \multicolumn{13}{|c|}{ Período 2080-2099 } \\
\hline & $5 \mathrm{~m}$ & $10 \mathrm{~m}$ & $15 \mathrm{~m}$ & $30 \mathrm{~m}$ & $45 \mathrm{~m}$ & $1 \mathrm{~h}$ & $2 \mathrm{~h}$ & $3 \mathrm{~h}$ & $4 \mathrm{~h}$ & $8 \mathrm{~h}$ & $14 \mathrm{~h}$ & $24 \mathrm{~h}$ \\
\hline GFCM21 & -3 & -2 & -3 & -2 & -2 & -1 & -3 & -3 & -4 & -5 & -4 & -4 \\
\hline HADCM3 & 13 & 14 & 13 & 14 & 14 & 15 & 14 & 13 & 11 & 11 & 12 & 12 \\
\hline INCM3 & 19 & 20 & 19 & 20 & 21 & 21 & 20 & 19 & 17 & 17 & 18 & 18 \\
\hline IPCM4 & 9 & 10 & 9 & 10 & 10 & 11 & 10 & 9 & 8 & 7 & 8 & 8 \\
\hline MPEH5 & 39 & 39 & 39 & 40 & 40 & 41 & 39 & 39 & 36 & 36 & 37 & 38 \\
\hline NCCCSM & 22 & 22 & 22 & 23 & 23 & 24 & 22 & 22 & 20 & 20 & 21 & 21 \\
\hline
\end{tabular}

INCM3 praticamente não apresentou alteração com a média dos desvios não ultrapassando os 3\%. O MPEH5 apresentou resultados superiores a $25 \%$.

No cenário mais pessimista A2 e primeiro período de projeção na Tabela 9, todos os modelos apresentaram em média resultados semelhantes com desvios na faixa de 9 a 14\% a exceção dos modelos MPEH5 e NCCCSM que apresentaram em média desvios superiores a $18 \%$.

Para o cenário mais otimista $\mathrm{B} 1$ no primeiro período de projeção, Tabela 10, os modelos GFCM21, HADCM3 e MPEH5 apresentaram em média resultados semelhantes com desvios na faixa de 9 a $15 \%$. O INCM3 praticamente não apresentou alteração com desvios menores que 3\%. No IPCM4 os desvios foram menores que 7\%. O
NCCCSM apresentou em média desvios superiores a $19 \%$.

No segundo período houve uma grande variabilidade entre os resultados dos modelos, para os cenários A1B intermediário e pessimista $\mathrm{A} 2$, com a média do desvios variando de negativo na faixa de $-4 \%$ a $24 \%$, Tabelas 8 e 9. Quando considerado o cenário B1, Tabela 10, a média dos desvios nos modelos GFCM2, HADCM3 e IPCM4 ficaram em média na faixa entre 6 e $14 \%$ e, nos modelos INCM3 e NCCCSM entre 14 e 18\%. Os maiores desvios ficaram por conta do MPEH5, em torno de $30 \%$.

Observa-se ao analisar os resultados, das Tabelas 8 a 10 , que alguns modelos apresentaram variabilidade maior em relação aos demais, entretanto a maioria dos modelos 
Tabela 10 - Média do desvio percentual médio cenário B1 por período nas diferentes durações.

\begin{tabular}{|c|c|c|c|c|c|c|c|c|c|c|c|c|}
\hline \multicolumn{13}{|c|}{ Período 2046-2065 } \\
\hline & $5 \mathrm{~m}$ & $10 \mathrm{~m}$ & $15 \mathrm{~m}$ & $30 \mathrm{~m}$ & $45 \mathrm{~m}$ & $1 \mathrm{~h}$ & $2 \mathrm{~h}$ & $3 \mathrm{~h}$ & $4 \mathrm{~h}$ & $8 \mathrm{~h}$ & $14 \mathrm{~h}$ & $24 \mathrm{~h}$ \\
\hline GFCM21 & 11 & 12 & 11 & 12 & 12 & 13 & 11 & 11 & 9 & 9 & 10 & 10 \\
\hline HADCM3 & 12 & 13 & 12 & 13 & 13 & 14 & 13 & 12 & 10 & 10 & 11 & 11 \\
\hline INCM3 & 1 & 2 & 1 & 2 & 3 & 3 & 2 & 1 & 0 & 0 & 0 & 1 \\
\hline IPCM4 & 5 & 6 & 5 & 6 & 6 & 7 & 6 & 5 & 4 & 3 & 4 & 5 \\
\hline MPEH5 & 13 & 14 & 13 & 14 & 15 & 15 & 14 & 13 & 12 & 11 & 12 & 13 \\
\hline NCCCSM & 20 & 20 & 20 & 21 & 21 & 21 & 20 & 20 & 20 & 18 & 18 & 19 \\
\hline \multicolumn{13}{|c|}{ Período 2080-2099 } \\
\hline & $5 \mathrm{~m}$ & $10 \mathrm{~m}$ & $15 \mathrm{~m}$ & $30 \mathrm{~m}$ & $45 \mathrm{~m}$ & $1 \mathrm{~h}$ & $2 \mathrm{~h}$ & $3 \mathrm{~h}$ & $4 \mathrm{~h}$ & $8 \mathrm{~h}$ & $14 \mathrm{~h}$ & $24 \mathrm{~h}$ \\
\hline GFCM21 & 7 & 7 & 7 & 8 & 8 & 9 & 7 & 7 & 5 & 5 & 6 & 6 \\
\hline HADCM3 & 10 & 10 & 10 & 11 & 11 & 12 & 10 & 10 & 8 & 8 & 9 & 9 \\
\hline INCM3 & 15 & 16 & 16 & 17 & 17 & 17 & 16 & 16 & 14 & 14 & 14 & 15 \\
\hline IPCM4 & 12 & 13 & 13 & 14 & 14 & 14 & 13 & 12 & 11 & 10 & 11 & 12 \\
\hline MPEH5 & 31 & 31 & 31 & 32 & 32 & 33 & 31 & 31 & 29 & 28 & 29 & 30 \\
\hline NCCCSM & 16 & 17 & 17 & 18 & 18 & 18 & 17 & 17 & 15 & 14 & 15 & 16 \\
\hline
\end{tabular}

indicou um aumento das precipitações sobre Porto Alegre. As diferenças de resultados são inerentes aos modelos matemáticos que representam o clima devido à complexidade entre as interações atmosféricas. De acordo com Valverde e Marengo (2010) as diferentes parametrizações, representativas dos processos físicos atmosféricos, tais como processos em superfície, convecção, camada limite, conteúdo de aerossóis, entre outros, à medida que forem aprimoradas reduzirão as incertezas.

\section{Conclusão}

O modelo LARS-WG se mostrou eficiente em simular as precipitações diárias em Porto Alegre para um clima atual, funcionando adequadamente tanto ao ajustar a distribuição do período seco quanto do período chuvoso. Também apresentaram bons ajustes os resultados na simulação das distribuições das chuvas diárias agregadas em níveis mensais.

$\mathrm{Na}$ avaliação da possível influência dos três cenários de clima futuro, estabelecidos pelo IPCC, sobre as relações IDF de Porto Alegre observam-se comportamentos diferentes para o período de 2046 a 2065 e para o período de 2080 a 2099. Os resultados encontrados para as projeções no primeiro período indicam que as simulações dos modelos são razoavelmente aplicáveis, sugerindo a utilização da curva IDF atual com uma majoração de 10 a $20 \%$ no dimensionamento de obras hidráulicas. Já para o segundo período de projeção em função da grande variabilidade nos resultados encontrados para os diferentes modelos não há confiança na aplicabilidade destas projeções.

\section{Referências}

BACK, A.J.; OLIVEIRA, J.L.R.; HENN, A.; Relações entre precipitações intensas de diferentes durações para desagregação da chuva diária em Santa Catarina. Revista Brasileira de Engenharia agrícola e Ambiental, v.16, p. 391398, 2012. Disponível em: <http://www.agriambi.com.br/ revista/v16n04/v16n04a09.pdf>. Acesso em: $01 \mathrm{dez} .2017$.

BELL, F.G. "Generalized rainfall - duration - frequency relationships" Journal of the Hydraulics Division - ASCE, v. 95, n. HY1, p. 311-27, 1969. apud CHEN, C. Rainfall Intensity duration - frequency formulas. Journal of Hydraulic Engineering - ASCE, v. 109, n. 12, p. 1603-21, 1983.

BENFICA, D. da C. Análise da aplicabilidade de padrões de chuva de projeto a Porto Alegre. 1999. 109 p. Dissertação (Mestrado Recursos Hídricos e Saneamento Ambiental) -Universidade Federal do Rio Grande do Sul, Porto Alegre (RS), 1999.

BERLATO, A.M.; CORDEIRO, A.P.; Sinais de mudanças climáticas globais e regionais, projeções para o século XXI e as tendências observadas no Rio Grande do Sul: uma revisão. Agrometeoros, v. 25, n. 2, p. 273-302, 2017.

BERTONI, J.C. E TUCCI, C.E.M. Precipitação, 3. Ed. In: TUCCI et al. (Orgs). Hidrologia: ciência e aplicação. Porto Alegre : Ed UFRGS/ABRH, 2004. p. 177-241.

BLAKELY, E.J. Urban Planning for Climate Change. Lincoln Institute of Land Policy Working Paper, 25p, 2007. Disponível em: <http://www.lincolninst.edu/sites/default/files/ pubfiles/1310_blakely_final.pdf>. Acesso em: $01 \mathrm{dez}$. 2017.

BLODGETT, J.C. Precipitation depth-duration and frequency characteristics for Antelop Valley, Mojave Desert, California. Sacramento, Calif.: US Dept. of Interior, U.S. Geological Survey. 33p, 1996. Disponível em: $<$ https://babel.hathitrust.org/cgi/pt? id=mdp. 39015037737999; view=1 up;seq=3>. Acesso em: 01 dez. 2017. 
CHEN, C. Rainfall Intensity - duration - frequency formulas. Journal of Hydraulic Engineering - ASCE, v. 109, n. 12, p. 1603-21, 1983. Disponível: <https://ascelibrary.org/doi/ pdf/10.1061/\%28ASCE\%290733-9429\%281983\%29109\% 3A12\%281603\%29>. Acesso em: 01 dez. 2017.

CHEN, J.; BRISSETTE, F.P.; LECONTE, R. WeaGETS - A Matlab-Based DailyScale Weather Generator for Generating Precipitation and Temperature. Procedia Environmental Sciences , v. 13, p. 2222-2235, 2012. <http://www. sciencedirect.com/science/article/pii/ S1878029612002125>. Acesso em: 01 dez. 2017.

CRUTCHER, H.L. A note on the possible misuse of the Kolmogorov-Smirnov test. Journal of Applied Meteorology, v. 14, p. 1600-1603, 1975. Disponível em: <http://journals. ametsoc.org/doi/pdf/10.1175/1520-0450(1975)014\% 3C1600:ANOTPM\%3E2.0.CO;2>. Acesso em: $01 \mathrm{dez}$. 2017.

DEP-IPH. Plano diretor de drenagem urbana: manual de drenagem urbana. Porto Alegre: Prefeitura Municipal; Departamento de Esgotos Pluviais, 2002. v. 2, 184 p, 2002.

GALVÃO, W.S.; MENESES, P.R. Avaliação dos sistemas de classificação e codificação das bacias hidrográficas brasileiras para fins de planejamento de redes hidrométricas. In: SIMPÓSIO BRASILEIRO DE SENSORIAMENTO REMOTO, 12. (SBSR), 2005, Goiânia. Anais... São José dos Campos: INPE, 2005. p. 2511-2518. Disponível em: $<$ http://marte.sid.inpe.br/col/ltid.inpe.br/sbsr/2004/ 11.21.01.26/doc/2511.pdf>

GOOSSE, H.; BARRIAT, P.Y.; LEFEBVRE, W.; LOUTRE, M. F.; ZUNZ, V. Introduction to climate dynamics and climate modelling. Livro online. Disponível em: <http:// www.climate.be/textbook/ebook.html>. Edition: Cambridge University Press. 2010. Acesso em: 01 dez. 2017.

HASSAN, Z.; HARUN, S. Impact of Climate Change on Rainfall over Kerian, Malaysia with Long Ashton Research Station Weather Generator (Lars-Wg). Malaysian Journal of Civil Engineering, v. 25, p. 33-44, 2013. Disponível em: <http://civil.utm.my/mjce/files/2014/04/Paper-4-251.pdf>. Acesso em: 01 dez. 2017.

HELSEL, D.R.; HIRSCH, R.M. Statistical Methods in Water Resourses. Ed. USGS, United States, 510p. 2002. <https:// pubs.usgs.gov/twri/twri4a3/pdf/twri4a3-new.pdf>. Acesso em: 01 dez. 2017.

MARCUZZO, F.F.N. Bacias hidrográficas e regiões hidrográficas do brasil: cálculo de áreas, diferenças e considerações. In: XXII Simpósio Brasileiro de Recursos Hídricos, 2017, Florianópolis. Anais... Porto Alegre: ABRH, 2017. p. 1-8. Disponível em: <http://rigeo.cprm.gov.br/jspui/bitstream/ doc/18492/1/2017_sbrh_bacias_hidrograficas_brasil_artigo.pdf $>$. Acesso em: 01 dez. 2017.

MARENGO, J.A. Mudanças Climáticas: Deteç̧ão e cenários futuros para o Brasil até o final do século XXI. In: CAVALCANTI et al. (Orgs). Tempo e Clima no Brasil. São Paulo: Ed Oficina de Textos, 2009. p. 407-420.

MCKAGUE, K.; RUDRA, R.; OGILVIE, J.; AHMED, I.; GHARABAGHI, B. (2005) Evaluation of Weather Generator ClimGen for Southern Ontario. Canadian Water Resources Journal , v. 30, p. 315-330. Disponível em:
$<$ http://www.tandfonline.com/doi/abs/10.4296/

cwrj3004315>. Acesso em: 01 dez. 2017.

MILLER, J.F.; FREDERICH, R.H.; TRACEY, R.J. Precipitation-frequency atlas of the western United States. Atlas 2, v.XI, California: 71p., 1973. Disponível em: <https:// permanent.access.gpo.gov/gpo46930/V.\%2011-California/ >. Acesso em: $01 \mathrm{dez} .2017$.

NAGHETTINI, M., PINTO, E. J. A. Hidrologia Estatística. Ed. CPRM, Belo Horizonte 552 p. 2007. <http://www.cprm. gov.br/publique/Hidrologia/Mapas-e-Publicacoes/Livro-\% 22Hidrologia-Estatistica\%22-981.html>. Acesso em: 01 dez. 2017.

OCCHIPINT, A.G.; SANTOS, P.M. Relações entre as precipitações máximas de um dia e de $\mathbf{2 4}$ horas na cidade de São Paulo. São Paulo: IAU/USP, 1966. 10p.

PBMC - Painel Brasileiro de Climáticas, 2014. Disponível em: $<$ http://www.pbmc.coppe.ufrj.br/pt/noticias/388-climatechanges-in-brazil-through-2100>. Acesso em: $01 \mathrm{dez}$. 2017.

PINTO, E. J.A. Metodologia para definição das equações Intensidade-Duração-Frequência do Projeto Atlas Pluviométrico. CPRM. Belo Horizonte. Mar, 2013. CD-ROM.

QIAN, B.; HAYHOE, H.; GAMEDA, S. Evaluation of the Stochastic Weather Generators LARS-WG and AAFC-WG for Climate Change Impact Studies. Climate Research. v. 29, p. 3-21, 2005. Disponível em: <http://www.int-res.com/ abstracts/cr/v29/n1/p3-21/>. Acesso em: 01 dez. 2017.

RACSKO, P.; SZEIDL, L.; SEMENOV, M. A serial approach to local stochastic weather models. Ecol Model v. 57 p. 27-41, 1991. Disponível em: <http://www.sciencedirect.com/science/article/pii/0304380091900534?via\%3Dihub>. Acesso em: 01 dez. 2017.

RIETVELD, M.R. (1978) A New Method for Estimating the Regression Coefficients in the Formula Relating Solar Radiation to Sunshine. Agricultural and Forest Meteorology, v. 19, p. 243-252. Disponível em: <http://www.sciencedirect.com/science/article/pii/0002157178900146?via \%3Dihub>. Acesso em: 01 dez. 2017.

SANDINK, D.; SIMINOVIC, S.P.; SCHARDONG, A.; SRIVASTAV, R. A decision support system for updating and incorporating climate change impacts into rainfall intensity-duration-frequency curves: Review of the stakeholder involvement process. Environmental Modelling e Software, v. 84, p. 193-209, 2016. Disponível em: <http:// www.sciencedirect.com/science/article/pii/ S1364815216302420?via\%3Dihub>. Acesso em: $01 \mathrm{dez}$. 2017.

SCHARDONG, A.; SIMONOVIC, S.P. Possíveis impactos de mudanças climáticas em curvas intensidade duração e frequência. In: XX SIMPÓSIO BRASILEIRODE RECURSOS HÍDRICOS -ABRH, 2013. Anais... Bento Gonçalves (RS), Brasil, 17 a 22 de novembro 2013. Disponível em: $<$ https://www.abrh.org.br/SGCv3/index.php?PUB=3\&ID=155\&PUBLICACAO=SIMPOSIOS $>.01 \mathrm{dez} .2017$.

SEMENOV, M.A.; BARROW, E.M. Use of a stochastic weather generator in the development of climate changes scenarios. Climatic Changes, Amsterdam, v.35, p. 397-414, 1997. Disponível em: <https://link.springer.com/article/10.1023/ A\%3A1005342632279>. Acesso em: 01 dez. 2017. 
SEMENOV, M.A.; BARROW, E.M. LARS-WG stochastic weather generator for use in climate impact studies. User manual version 3.0. 2002. Disponível em: <http://resources.rothamsted.ac.uk/sites/default/files/groups/mas-models/download/LARS-WG-Manual.pdf>. Acesso em: 01 dez. 2017.

SEMENOV, M.A. Simulation of extreme weather events by a stochastic weather generator. Climate Research, v. 35, p. 203-212, 2008. Disponível em: <http://www.int-res.com/ articles/cr2007/35/c035p203.pdf $>$. Acesso em: 01 dez. 2017.

SEMENOV M.A.; STRATONOVITCH, P. Use of Multi-Model Ensembles from Global Climate Models for Assessment of Climate Change Impacts. Climate Research , v.41, p. 114, 2010. Disponível em: <http://www.int-res.com/articles/ cr_oa/c041p001.pdf>. Acesso em: 01 dez. 2017.

STOCKLE, C.O.; DONATELLI, M.; NELSON, R. CropSyst, a Cropping Systems Simulation Model. European Journal of Agronomy, v. 18, p. 289-307, 2003. Disponível em: $<$ https://www.sciencedirect.com/science/article/pii/ S1161030102001090>. Acesso em: 01 dez. 2017.
TODESCHINI, S. Trends in long daily rainfall Lombardia (northern Italy) affecting urban stormwater control. International Journal of Climatology. v.32, p. 900-919, 2012. Disponível em: <http://onlinelibrary.wiley.com/doi/ 10.1002/joc.2313/pdf>. Acesso em: 01 dez. 2017.

VALVERDE, M.C.; MARENGO, J.A. Mudanças na Circulação Atmosférica sobre a América do Sul para Cenários Futuros de Clima Projetados pelos Modelos Globais do IPCC AR4. Revista Brasileira de Meteorologia, v. 25, n. 1 p. 125145, 2010. Disponível em: <http://www.scielo.br/pdf/ rbmet/v25n1/v25n1a11.pdf $>$. Acesso em: 01 dez. 2017.

WESCHENFELDER, A.B.; PICKBRENNER, K.; PINTO, E.J. A. Atlas Pluviométrico do Brasil. Equações Intensidade-Duração-Frequência. Município: Porto Alegre, Estação Pluviográfica: Porto Alegre, Código 03051011. CPRM/SGB. Porto Alegre. 2015. <http://rigeo.cprm.gov. br/jspui/bitstream/doc/18700/1/Relatorio_idf_pa.pdf $>$. Acesso em: 01 dez. 2017.

This is an Open Access article distributed under the terms of the Creative Commons Attribution Non-Commercial License which permits unrestricted non-commercial use, distribution, and reproduction in any medium provided the original work is properly cited. 